ORIGINAL ARTICLE

\title{
Topical adrenaline and cocaine gel for anaesthetising children's lacerations. An audit of acceptability and safety
}

\author{
D W G Kennedy, Z Shaikh, M J Fardy, R J Evans, StJ V Crean
}

Emerg Med J 2004;21:194-196. doi: 10.1136/emj.2003.010108

See end of article for authors' affiliations

Correspondence to Mr DW G Kennedy, Maxillofacial Unit, University Hospital of Wales, Heath, Cardiff CF64 ISP, UK; dwgkennedy@aol.com

Accepted for publication 7 August 2003

\begin{abstract}
Objectives: (1) To assess the acceptability of a gel solution of adrenaline (epinephrine) (1 in 2000) and cocaine (5\%) for anaesthetising children's facial lacerations to the child, parent, and operator. (2) To assess the safety of the current protocol.

Setting: The emergency unit of a large university hospital.

Methods: All patients who were treated with topical adrenaline and cocaine (topAC) gel over a six month period were entered into a prospective audit $(n=75)$. Patient details, the nature and cause of the injury, and any treatment carried out were all recorded. The acceptability to children over 3 years of age, was assessed by the use of the Wong Baker face scale, in which 0 represents "no hurt" and 5 represents "hurts worst". The acceptability to both the parent and the operator was assessed by the use of a 0 to 9 Likert scale, where 0 represented "very acceptable" and 9 represented "not at all" acceptable.

Results: (1) Children aged 3 years or older graded their pain during the procedure as having a mean value of 1.17 on the Wong Baker (0 to 5) scale. Parents graded acceptability on the Likert scale (0 to 9) with a mean score of 1.13. Operators using the same grading system, recorded a mean score of 1.75. (2) No toxic side effects were seen but the protocol was updated in line with evidence.

Conclusions: Topical adrenaline and cocaine is an effective anaesthetic for suturing children's facial lacerations and is acceptable to child, parent, and operator alike.
\end{abstract}

S oft tissue injuries in children, and their management, can be stressful for the patient, the child's parents, and for the staff dealing with them. Physical restraint of uncooperative children should be discouraged and sedation techniques carry risks that may not be appropriate, especially if carried out by non-anaesthetists. ${ }^{1}$ To provide an alternative to injecting local anaesthetics, Pryor et al introduced a topical anaesthetic solution for laceration repair in $1980 .^{2}$ The original solution appears to have been devised empirically and contained tetracaine $(0.5 \%)$, adrenaline (epinephrine) (1:1000), and cocaine (11.8\%), and it was given the acronym TAC. The gel used in this study, which contains adrenaline 1 in 2000 and cocaine 5\%, (topAC) has evolved from Pryor's original TAC liquid solution. Several papers have shown that the component concentrations could be reduced to improve safety. ${ }^{3-5}$ The gel is not used on mucous membranes, or in proximity to them (as absorption is far more rapid), nor on the pinna, fingers, toes, nose, penis, on abrasions or burns, or where tissue viability is in question. A gel very similar to the one used in this audit was compared with $1 \%$ lignocaine (lidocaine) infiltration by Kendall et al and was found to be significantly more acceptable to the patient. ${ }^{6}$ There are several papers that have compared various concentrations of TAC against infiltration of local anaesthetic and shown that TAC provides equivalent anaesthesia for suturing facial lacerations but is more acceptable to the children. ${ }^{27-9}$

The aims of this paper were to assess the acceptability of topAC gel to children with facial lacerations, to their parents, and to the staff carrying out the laceration repair. We have also assessed the use of topAC gel in terms of safety of our current protocol and against published literature with a view to revising the protocol where necessary.

\section{METHOD}

This audit was carried out in the emergency unit of a university teaching hospital that sees 25000 new child attendees per annum. There was a printed protocol for the use of topAC gel that had been in place since the gel was introduced into the emergency unit in April 2000. Children attending the paediatric emergency unit with wounds that required sutures were referred to the maxillofacial unit for repair. All children who were referred and were treated with topAC gel from December 2001 to April 2002 were entered into the audit. A small number of wounds requiring, for example, general anaesthesia for cleaning then repair were not treated with topAC and therefore not included in the audit. All laceration repairs were carried out by senior house officers in maxillofacial surgery. The audit was conducted by the SHO completing an audit form immediately after completion of treatment. Demographic details of the patients were collected as well as descriptive details of the size, shape, and site of the wound. The treatment carried out was recorded including the number of sutures and whether supplemental local anaesthetic or inhalational analgesia was used. It was recorded whether or not the parent stayed in the room and whether any form of restraint was used, including parental holding.

Acceptability to the patient was assessed by the use of Wong Baker faces, which are a well validated method of assessing pain in children. ${ }^{10}$ This scale can be used in children of 3 years and older and they were asked to indicate their "hurt" by indicating the appropriate face numbered 0 to 5 . Parents and the operator were asked to grade acceptability of the procedure on a 0 to 9 Likert scale where 0 represented "very acceptable", 4 to 5 was "moderate", and 9 was equivalent to "not at all" acceptable. The parents were also asked a direct question- "Was this procedure acceptable for your child?" and the answer recorded.

\section{RESULTS}

Fifty one (68\%) of the group were boys, 24 (32\%) were girls. The age range of the children was from 9 months to 16 years old but most children were aged 4 to 8 years old (59\%). 


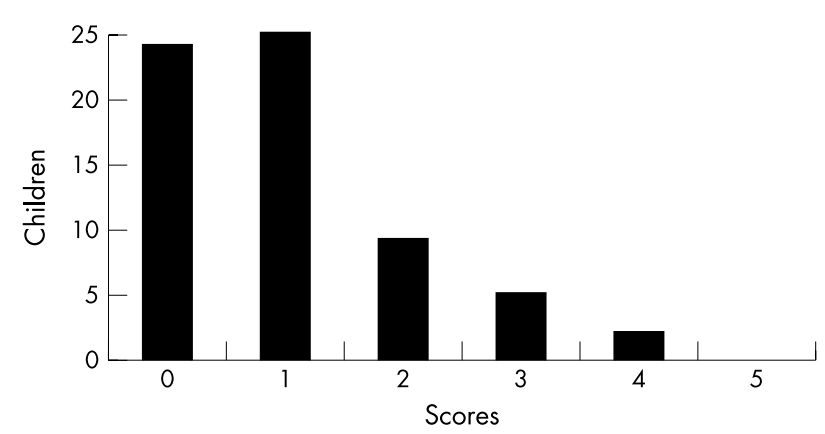

Figure 1 Wong Baker face scores for children. Reprinted by permission of Mosby Inc.

The commonest site of a laceration treated with topAC was the forehead (55\%), followed by eyebrow (16\%), and chin (16\%). The size of the lacerations ranged from $7 \mathrm{~mm}$ to $45 \mathrm{~mm}$. The mean size was $19.8 \mathrm{~mm}$ (SD $8.6 \mathrm{~mm}$ ). Sixty one per cent required deep sutures and 19\% required three or more deep sutures.

No inhalational analgesia was used, $66(88 \%)$ were treated with topAC alone, the other 9 (12\%) had supplemental local anaesthetic. Children over 3 years old were asked to indicate which of the Wong Baker face matched their "hurt" during the laceration repair, there were 65 children in this group and $49(75 \%)$ graded the experience as either "no hurt" or "hurts little bit"; the results are shown in figure 1.

There was at least one parent present with their child during the wound repair and so they were able to assess the procedure. Both parents and operators graded acceptability very highly. On the scale used, 0 was equivalent to very acceptable and $59(79 \%)$ parents graded either 0 or 1 . The operators also graded acceptability of the procedure highly, $59(79 \%)$ graded either 0 or 1 on the Likert scale, as shown in figure 2 .

No doctor or nurse restraint was used to treat the patients in this audit, nor was any improvised or commercial restraint device used. However, 22 children were held by their parents during suturing, the average age of these children was 3.9 years compared with an average age of 7.6 years in the group that were not held by their parents. Of the 22 parents that held their children for the procedure, 17 still graded the procedure as 0,1 , or 2 on the Likert scale.

The direct question "Was this procedure acceptable for your child" was asked of the parents and 94.7\% answered that is was. Of the four parents who answered that it was not acceptable, the procedure had been scored poorly acceptable by the child, operator, and parent previously.

\section{SAFETY OF THE PROTOCOL}

There were no adverse effects related to the absorption of the constituents of the gel during the time of this audit. Both cocaine and adrenaline are toxic in overdose and the clinician should be aware of the signs of toxicity and management of the overdosed patient. The first papers describing the use of various TAC liquid solutions, all described giving the dose that was determined by the length of the laceration, ${ }^{211}{ }^{12}$ the maximum dose being decided either by a predetermined volume of solution that was allowed to be used, or by a predetermined maximal wound size that could be treated. The protocol that was followed in this hospital broadly followed this pattern. It emphasised the safe application of the gel and the avoidance of areas supplied by an end artery, such as fingers and toes and the absolute avoidance of mucous membranes and described how it should be applied. The maximal dose to be used was $5 \mathrm{ml}$ of the gel, containing a total of $250 \mathrm{mg}$ of cocaine. It became clear in this audit that the gel was being used on very small children and that our dose should be determined by weight rather than laceration size. A Medline search was carried out to determine evidence for determining a dose regimen based on weight. The first paper to describe a weight dependent dose was by Hegenberth et $a l^{7}$ in 1990, they recommended a maximal dose of $10.6 \mathrm{mg} / \mathrm{kg}$. Since then articles by Cote, ${ }^{13}$ Sectish, ${ }^{14}$ and the American Academy of Pediatrics, Committee on Drugs $^{15}$ have all recommended a maximal dose of $6 \mathrm{mg}$ of cocaine per kilogram. There are no recommendations in the literature about maximal doses of adrenaline, however using the smallest possible dose of cocaine will automatically decrease the amount of adrenaline used. The protocol for use in this hospital now has $6 \mathrm{mg} / \mathrm{kg}$ as the maximal dose and we believe it is now as safe as possible. The whole protocol is reproduced on the journal web site (http://www.emjonline. com/supplemental). There have been several studies carried

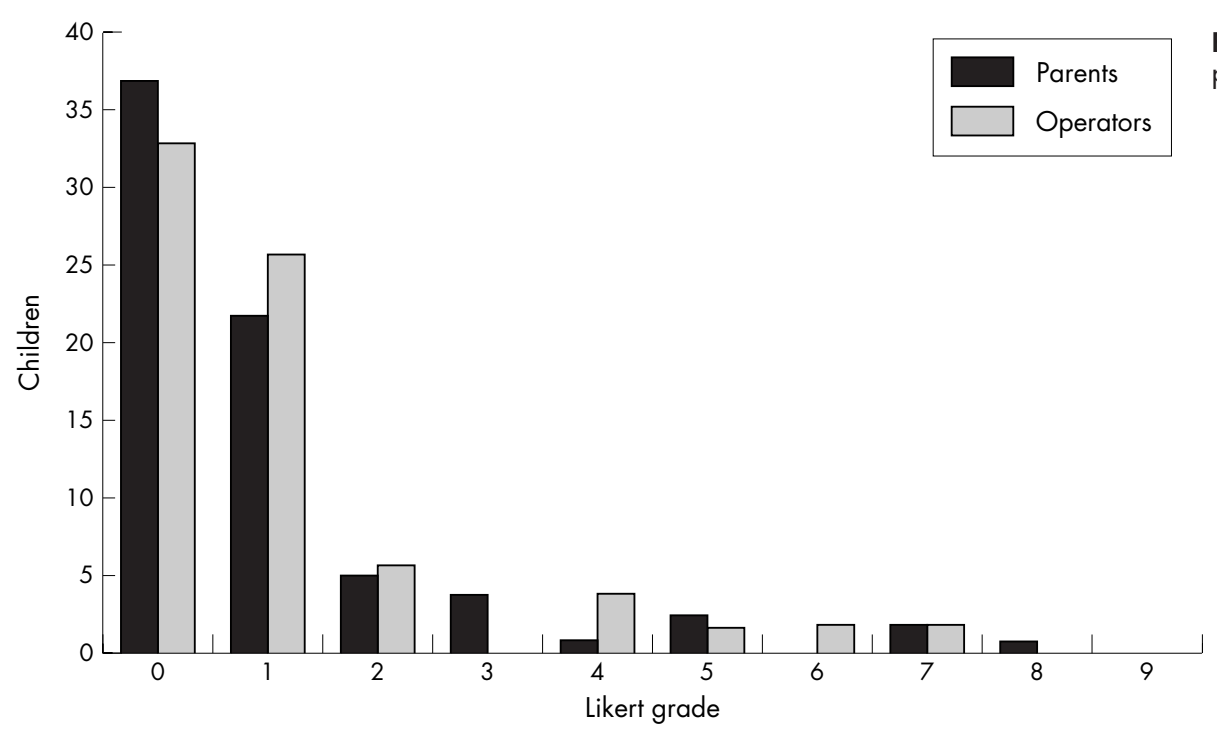

Figure 2 Grades of acceptability to parents and operator. 
out to establish a non-cocaine alternative topical anaesthetic, ${ }^{16}$ based on strong lignocaine, which will also have to be prescribed in a weight dependent method. An obvious advantage of this type of agent would be avoidance of controlled drug restrictions. More research will establish which topical anaesthetic agent is the most effective and safest.

\section{DISCUSSION}

We believe that this audit lends support to the use of topical adrenaline and cocaine gel as a suitable agent for anaesthetising children's lacerations before suturing. Its use is acceptable to the child, the operator, and the parent. The keys to avoiding toxic concentrations of cocaine absorption are firstly to prevent mucosal exposure and secondly to limit the total amount of cocaine used. Over the 22 years since the introduction of the original TAC solution, there have been two deaths reported. Both of these followed severe misuse of the solution, in one case a huge dose of the liquid ran into the nose $\mathrm{e}^{17}$ and in the other TAC was applied directly onto the tongue. ${ }^{18}$ The use of a gel form, which is much less likely to run, the use of far smaller yet efficacious doses, but primarily the adherence to a safe protocol will prevent any such disasters occurring again.

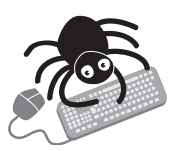

The protocol is available to view on the journal web site (http://www.emjonline.com/supplemental).

\section{Authors' affiliations}

D W G Kennedy, Z Shaikh, M J Fardy, StJ V Crean, Maxillofacial Unit, University Hospital of Wales, Heath, Cardiff, UK

R J Evans, Emergency Unit, University Hospital of Wales

\section{REFERENCES}

1 Scottish Intercollegiate Guidelines Network (SIGN). Safe sedation of children undergoing diagnostic and therapeutic procedures. SIGN publication no 54 . Edinburgh: SIGN, 2002.

2 Pryor GJ, Kilpatrick WR, Opp DR. Local anaesthesia in minor lacerations: topical TAC vs lidocaine infiltration. Ann Emerg Med 1980;9:568-71.

3 Bonadio WA, Wagner V. Half-strength TAC topical anaesthetic; for selected dermal lacerations. Clin Pediatr 1988;27:495-8.

4 Bonadio WA, Wagner V. Efficacy of tetracaine-adrenaline-cocaine topical anaesthetic without tetracaine for facial laceration repair in children. Pediatrics 1990;86:856-7.

5 Bonadio WA, Wagner V. Adrenaline-cocaine gel topical anaesthetic gel for dermal laceration repair in children. Ann Emerg Med 1992;21:1435-8.

6 Kendall JM, Charters A, McCabe SE. Topical anaesthesia for children's anaesthesia: an acceptable approach? J Accid Emerg Med 1996;13:1 19-29.

7 Hegenbarth MA, Altieri MF, Hawk WH, et al. Comparison of topical tetracaine, adrenaline, and cocaine anaesthesia with lidocaine infiltration for repair of lacerations in children. Ann Emerg Med 1990;19:63-7.

8 Anderson $A B$, Colecchi C, Baronoski R, et al. Local anesthetic in pediatric patients: Topical TAC versus lidocaine. Ann Emerg Med 1990;19:519-22.

9 Smith GA, Strausbaugh SD, Harbeck-Weber C, et al. Comparison of topical anesthetics with lidocaine infiltration during laceration repair in children. Clin Pediatr 1997;36:17-23.

10 Wong DL, Hockenberry-Eaton M, Wilson D, et al. Wong's essentials of paediatric nursing. 6th edn. St Louis: Mosby, 2001:1301.

11 Cannon CR, Chouteau S, Hutchison K. Topically applied tetracaine, adrenaline, and cocaine in the repair of traumatic wounds of the head and neck. Otolaryngol Head Neck Surg 1989;100:78-9.

12 Nichols FC, Mucha P, Farnell MB. TAC topical anaesthesia and minor skin lacerations. Resident Staff Physician 1987;33:59-64.

13 Cote CJ. Sedation for the pediatric patient. A review. Pediatr Clin North Am 1994:41:31-58

14 Sectish TC. Use of sedation and local anaesthesia to prepare children for procedures. Am Fam Physician 1997;55:909-16.

15 American Academy of Pediatrics, Committee on Drugs. Alternative routes of drug administration-advantages and disadvantages. Pediatrics 1997; 100:143-52.

16 Bush S. Is cocaine needed in topical anaesthesia? Emerg Med J 2002;19:418-22.

17 Dailey RH. Fatality secondary to misuse of TAC solution. Ann Emerg Med 1988;17:159-60.

18 Jacobsen S. Errors in emergency practice. Emerg Med 1987;19:109. 